\title{
Acute Effects of Preventing Heart Failure by Sodium- Glucose Cotransporter 2 Inhibitors
}

\author{
Hidekatsu Yanai
}

\section{To the Editor}

EMPA-REG OUTCOME showed a significantly lower rate of hospitalization for heart failure by $35 \%$ than placebo [1]. In the CANVAS program [2], there was a significantly lower rate of hospitalization for heart failure in the canagliflozin group (33\% relative risk reduction) than placebo. In DECLARETIMI 58, dapagliflozin resulted in a lower rate of hospitalization for heart failure (hazard ratio: $0.73 ; 95 \%$ confidence inter$\operatorname{val}(\mathrm{CI}): 0.61-0.88)$ [3].

A significant difference in hospitalization for heart failure between sodium-glucose cotransporter 2 inhibitors (SGLT2i) and placebo immediately after the start of the trial [1-3] made us think of acute and chronic effects of reducing heart failure by SGLT2i [4]. An early decline of estimated glomerular filtration rate (eGFR) from the start of SGLT2i and a subsequent gradual increase in eGFR were observed in EMPA-REG OUTCOME trial and our previous study $[5,6]$. The phase showing a decline of eGFR may indicate acute effects of SGLT2i. Osmotic diuresis and increased urinary sodium excretion may induce body fluid depletion, which may result in reducing blood pressure and preventing the development of heart failure by acting like diuretics (Fig. 1) [4].

In our previous study, an increase of hematocrit levels in the B-type natriuretic peptide (BNP)-increased group was significantly smaller than the BNP-decreased group [7]. The change in plasma BNP levels was negatively and significantly correlated with change in hematocrit levels. The eGFR decreased in the BNP-decreased group, and eGFR increased in the BNP-increased group. The change in plasma BNP levels was positively and significantly correlated with change in eGFR. Serum blood urea nitrogen (BUN) increased in the BNP-decreased group, and BUN decreased in the BNP-increased group. These results support a significant contribution of SGLT2i-induced body fluid depletion to prevention of heart failure in the early phase [7]. Chronic effects of SGLT2i for prevention of heart failure include improvement of myocardial energetics, reduction of al-

Manuscript submitted August 6, 2021, accepted August 13, 2021

Published online August 31, 2021

Department of Diabetes, Endocrinology and Metabolism, National Center for Global Health and Medicine Kohnodai Hospital, 1-7-1 Kohnodai, Ichikawa, Chiba 272-0034, Japan. Email: dyanai@hospk.ncgm.go.jp

doi: https://doi.org/10.14740/cr1315 buminuria, suppression of eGFR decline, reduced sympathetic overactivity and anti-atherosclerotic effects (Fig. 1) [4, 8, 9].

Recent randomized controlled trials (RCTs), DAPA-HF and EMPEROR-Reduced showed that reduced hospitalization for heart failure with administration of SGLT2i to non-diabetic patients with heart failure can better explain the acute effects of SGLT2i on improving heart failure [10, 11]. I calculated the risk reduction of hospitalization for heart failure at 6 months after the start of SGLT2i (regarded as acute phase) by using the cumulative incidence of hospitalization for heart failure in the pooled SGLT2i group and the placebo group (Fig. 2). All patients studied in EMPA-REG and CANVAS Program had type 2 diabetes. In both studies, SGLT2i significantly reduced the risk of hospitalization for heart failure during an early phase. DAPA-HF and EMPEROR-Reduced included over $50 \%$ of non-diabetic patients. Risk reduction of the hospitalization for heart failure in DAPA-HF and EMPEROR-Reduced was smaller than that in EMPA-REG and CANVAS Program (Fig. 2).

SGLT2 is the major cotransporter involved in reabsorption of filtered glucose in the proximal tubule of the kidney. In type 2 diabetes, an increased amount of glucose is filtered by the kidneys and SGLT2 is upregulated, leading to increased glucose absorption and worsening hyperglycemia [12]. SGLT2 $\mathrm{i}$ cause osmotic diuresis and calorie leakage into the urine; therefore, the benefits of SGLT2i could include blood pressure lowering and weight control [12]. Therefore, acute effects of SGLT2i such as osmotic diuresis, increased sodium excretion and reduced blood pressure may be greater in diabetic patients than in non-diabetic patients, which can explain the smaller risk reduction of the hospitalization for heart failure in DAPAHF and EMPEROR-Reduced as compared with that in EMPAREG and CANVAS Program. Smaller reduction of systolic blood pressure in DAPA-HF $(-1.27 \mathrm{~mm} \mathrm{Hg})$ and EMPERORReduced $(-0.7 \mathrm{~mm} \mathrm{Hg})$ as compared with that in EMPA-REG $(-3$ to $-4 \mathrm{~mm} \mathrm{Hg}$ ) and CANVAS Program $(-3.93 \mathrm{~mm} \mathrm{Hg})$ supports my hypothesis.

In conclusion, osmotic diuresis and increased urinary sodium excretion may induce body fluid depletion and reduction of blood pressure, which may be acute effects of preventing heart failure by SGLT2i.

\section{Acknowledgments}

I thank the staffs of the Division of Research Support, National Center for Global Health and Medicine Kohnodai Hospital. 


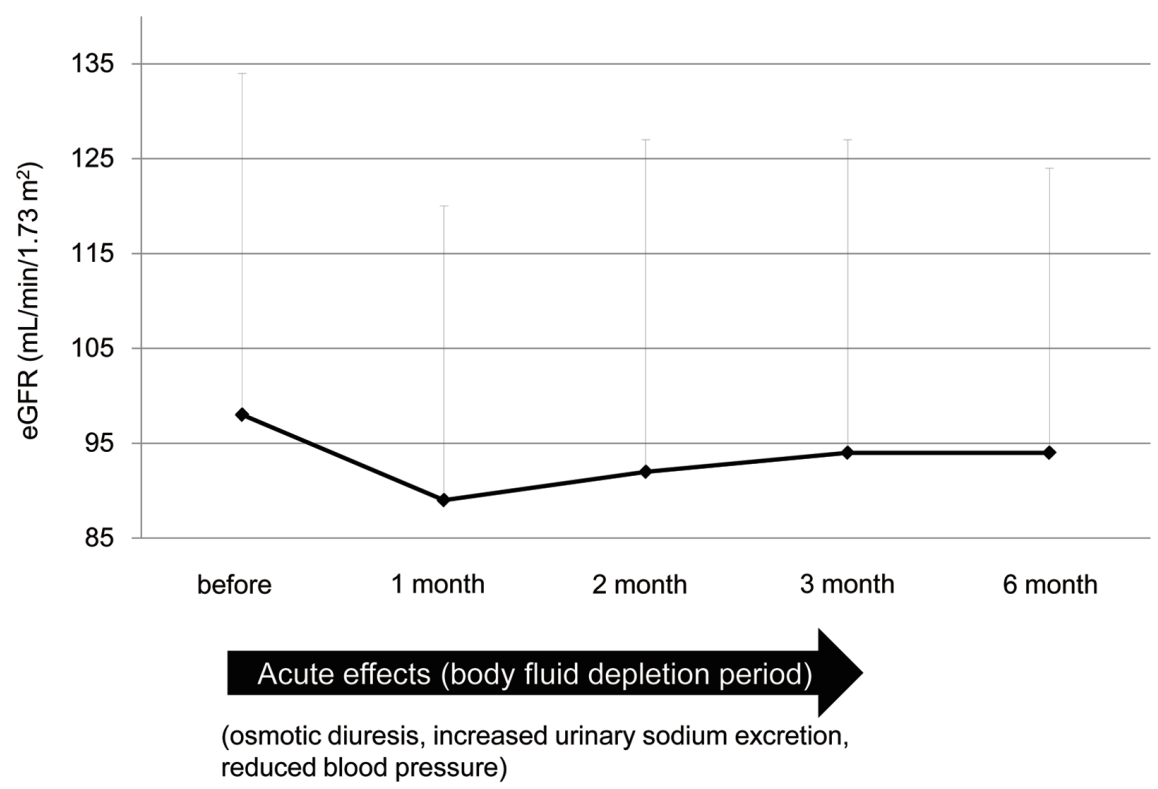

(osmotic diuresis, increased urinary sodium excretion duced blood pressure)

\section{Chronic effects}

(improvement of myocardial energetics, improvement of cardio-renal association, reduced sympathetic overactivity, anti-atherosclerotic effects)

Figure 1. Acute and chronic effects of preventing heart failure by sodium-glucose cotransporter 2 inhibitors (SGLT2i). The change of estimated glomerular filtration rate (eGFR) was made by modification of our previous study [6]

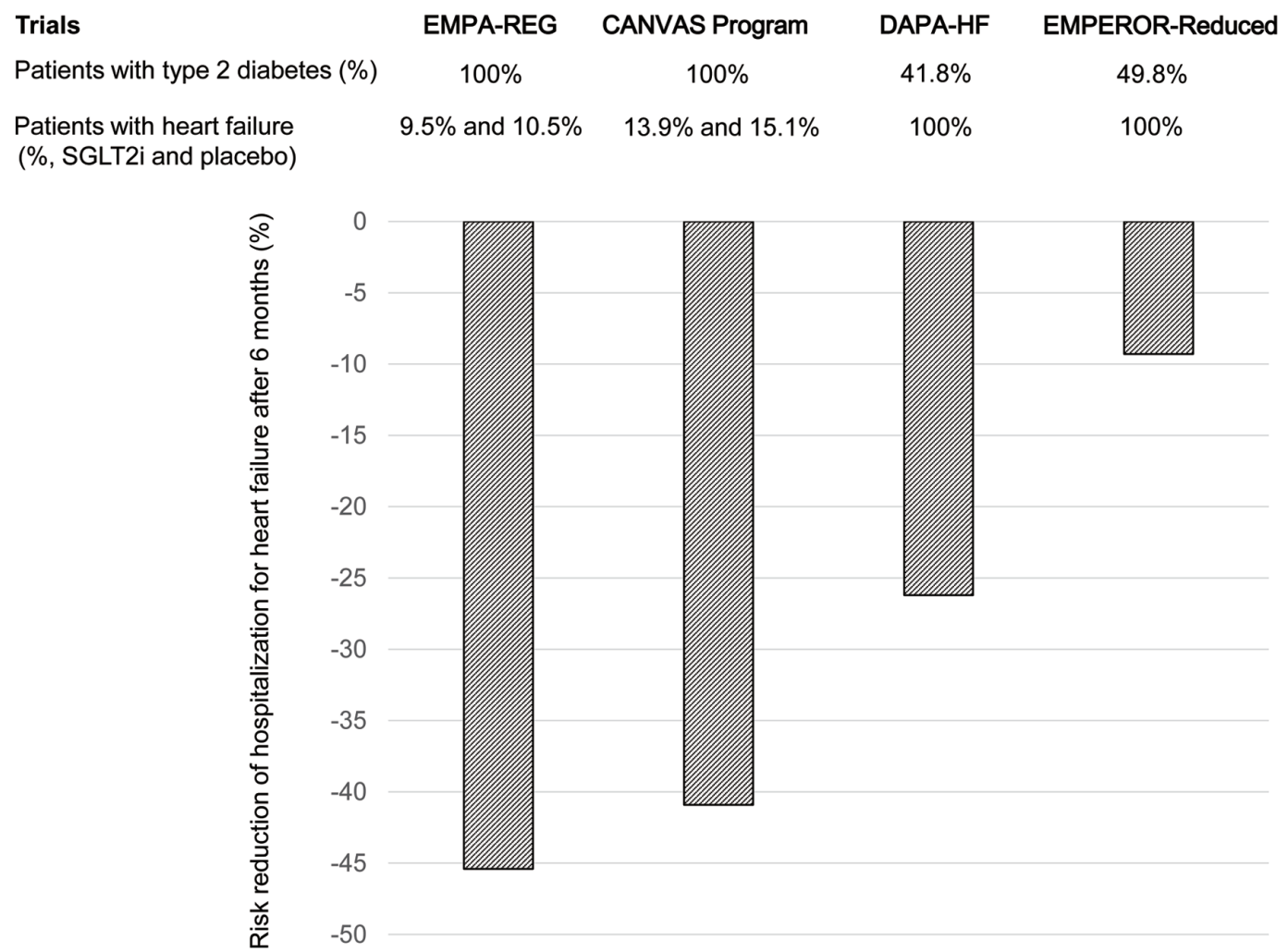

Figure 2. Risk reduction of the hospitalization for heart failure at 6 months after the start of sodium-glucose cotransporter 2 inhibitors (SGLT2i) in randomized controlled trials (RCTs) which included non-diabetic patients or not. 


\section{Financial Disclosure}

Author has no financial disclosures to report.

\section{Conflict of Interest}

The author declares that he has no conflict of interest concerning this article.

\section{Informed Consent}

Not applicable.

\section{Author Contributions}

HY designed the research, and collected and analyzed data. HY wrote and approved the final paper.

\section{Data Availability}

The data supporting the findings of this study are available from the corresponding author upon reasonable request.

\section{References}

1. Zinman B, Wanner C, Lachin JM, Fitchett D, Bluhmki E, Hantel S, Mattheus M, et al. Empagliflozin, cardiovascular outcomes, and mortality in type 2 diabetes. N Engl J Med. 2015;373(22):2117-2128.

2. Neal B, Perkovic V, Mahaffey KW, de Zeeuw D, Fulcher G, Erondu N, Shaw W, et al. Canagliflozin and cardiovascular and renal events in type 2 diabetes. N Engl J Med.
2017;377(7):644-657.

3. Wiviott SD, Raz I, Bonaca MP, Mosenzon O, Kato ET, Cahn A, Silverman MG, et al. Dapagliflozin and cardiovascular outcomes in type 2 diabetes. N Engl J Med. 2019;380(4):347-357.

4. Yanai H. Sodium-glucose cotransporter 2 inhibitors and death and heart failure in type 2 diabetes. Ann Transl Med. 2017;5(23):470.

5. Wanner C, Inzucchi SE, Lachin JM, Fitchett D, von Eynatten M, Mattheus M, Johansen OE, et al. Empagliflozin and progression of kidney disease in type 2 diabetes. N Engl J Med. 2016;375(4):323-334.

6. Yanai $\mathrm{H}$, Katsuyayama $\mathrm{H}$. A Possible mechanism for renoprotective effect of sodium-glucose cotransporter 2 inhibitor: elevation of erythropoietin production. J Clin Med Res. 2017;9(2):178-179.

7. Yanai $\mathrm{H}$, Adachi $\mathrm{H}$, Hakoshima M. Understanding of hypertension and heart failure in patients with type 2 diabetes by studying effects of sodium-glucose cotransporter 2 inhibitors on plasma B-type natriuretic peptide levels. J Clin Hypertens (Greenwich). 2018;20(2):411412.

8. Yanai H. Sodium-glucose cotransporter 2 inhibitors for heart failure. J Endocrinol Metab. 2017;7(3):5-76.

9. Sano M. A new class of drugs for heart failure: SGLT2 inhibitors reduce sympathetic overactivity. J Cardiol. 2018;71(5):471-476.

10. McMurray JJV, Solomon SD, Inzucchi SE, Kober L, Kosiborod MN, Martinez FA, Ponikowski P, et al. Dapagliflozin in patients with heart failure and reduced ejection fraction. N Engl J Med. 2019;381(21):1995-2008.

11. Packer M, Anker SD, Butler J, Filippatos G, Pocock SJ, Carson P, Januzzi J, et al. Cardiovascular and renal outcomes with empagliflozin in heart failure. N Engl J Med. 2020;383(15):1413-1424.

12. Ferrannini E, Solini A. SGLT2 inhibition in diabetes mellitus: rationale and clinical prospects. Nat Rev Endocrinol. 2012;8(8):495-502. 